\title{
Development of a Risk Score to Predict Detection of Metastasized or Locally Advanced Perihilar Cholangiocarcinoma at Staging Laparoscopy
}

\author{
Robert J. S. Coelen, MD', Anthony T. Ruys, MD, PhD ${ }^{1}$, Jimme K. Wiggers, MD, PhD ${ }^{1}$, Chung Y. Nio, MD $^{2}$, \\ Joanne Verheij, MD, $\mathbf{P h D}^{3}$, Dirk J. Gouma, MD, PhD ${ }^{1}$, Marc G. H. Besselink, MD, PhD ${ }^{1}$, \\ Olivier R. C. Busch, MD, PhD ${ }^{1}$, and Thomas M. van Gulik, MD, $\mathbf{P h D}^{1}$ \\ ${ }^{1}$ Department of Surgery, Academic Medical Center, Amsterdam, The Netherlands; ${ }^{2}$ Department of Radiology, Academic \\ Medical Center, Amsterdam, The Netherlands; ${ }^{3}$ Department of Pathology, Academic Medical Center, Amsterdam, The \\ Netherlands
}

\begin{abstract}
Background. Nearly half of patients with perihilar cholangiocarcinoma (PHC) have incurable tumors at laparotomy. Staging laparoscopy (SL) potentially detects metastases or locally advanced disease, thereby avoiding unnecessary laparotomy. However, the diagnostic yield of SL has decreased with improved imaging in recent years. Objective. The aim of this study was to identify predictors for detecting metastasized or locally advanced PHC at SL and to develop a risk score to select patients who may benefit most from this procedure.

Methods. Data of patients with potentially resectable PHC who underwent SL between 2000 and 2015 in our center were retrospectively analyzed. Multivariable logistic regression analysis was used to identify independent predictors and to develop a preoperative risk score.

Results. Unresectable PHC was detected in 41 of 273 patients undergoing SL (yield $15 \%$ ). Overall sensitivity of SL was $30 \%$, with highest sensitivity for detecting peritoneal metastases $(73 \%)$. Preoperative imaging factors that were independently associated with unresectability at SL were tumor size $\geq 4.5 \mathrm{~cm}$, bilateral portal vein
\end{abstract}

Electronic supplementary material The online version of this article (doi:10.1245/s10434-016-5531-6) contains supplementary material, which is available to authorized users.

(C) The Author(s) 2016. This article is published with open access at Springerlink.com

First Received: 2 March 2016;

Published Online: 1 September 2016

R. J. S. Coelen, MD

e-mail: r.j.coelen@amc.nl involvement, suspected lymph node metastases, and suspected (extra)hepatic metastases on imaging without the possibility of diagnosis by percutaneous- or endoscopic ultrasound-guided biopsy. The derived preoperative risk score showed good discrimination to predict unresectability (area under the curve $0.77,95 \%$ confidence interval 0.68-0.86) and identified three subgroups with a predicted low-risk of $7 \%(N=203$ patients $)$, intermediate-risk of $21 \%(N=39)$, and high-risk of $58 \%(N=31)$.

Conclusions. A selective approach for SL in PHC is recommended since the overall yield is low. The proposed preoperative risk score is useful in selecting patients for SL.

Perihilar cholangiocarcinoma (PHC) is a rare disease with a dismal prognosis for which radical resection remains the only curative treatment. ${ }^{1}$ Unfortunately, nearly half of patients subjected to laparotomy have unresectable tumors, despite extensive preoperative radiological staging., ${ }^{2,3}$ Staging laparoscopy (SL) in addition to imaging prior to exploratory laparotomy may detect small liver metastases or peritoneal metastases, thereby avoiding unnecessary laparotomy. However, the true additional diagnostic value of this procedure remains unclear, with varying results reported in the literature. ${ }^{4}$ Currently, the routine use of SL in preoperative staging of $\mathrm{PHC}$ is being questioned as the majority of studies from recent years have shown a diagnostic yield below $20 \%$ and a sensitivity to detect unresectable disease lower than $60 \% .^{5-9}$

As the routine use of SL in PHC patients does not seem justified, a selective approach to identify patients who may benefit most from this procedure seems warranted. ${ }^{5}$ There 
is currently no evidence for adequate selection criteria for SL in PHC patients as most studies are hampered by small sample size, resulting in low predictive power. The aim of this study was therefore to identify predictors of unresectable disease at SL and develop a preoperative risk score in a large cohort of PHC patients treated in a single center specializing in the management of PHC.

\section{METHODS}

\section{Study Population and Selection}

Consecutive patients with suspected $\mathrm{PHC}^{10}$ who were seen at the Academic Medical Center (AMC) in Amsterdam between May 2000 and July 2015 were identified from a prospectively maintained database. Exclusion criteria were SL for gallbladder carcinoma, and distal or intrahepatic cholangiocarcinoma.

All patients and radiological scans were discussed in a multidisciplinary, hepatopancreatobiliary (HPB) team meeting. In the event of suspected distant lymph node or organ metastases, percutaneous- or endoscopic ultrasound (EUS)-guided biopsy was attempted in order to confirm metastatic disease. When tumors were considered potentially resectable, SL was planned early in the preoperative workup in all patients with Bismuth-Corlette (BC) type 3-4 tumors. Laparoscopy for BC type 1-2 tumors was conducted more selectively according to the surgeon's preference. Laparoscopy was also performed in the event of suspicious metastatic lesions on imaging for which diagnosis by percutaneous- or EUS-guided biopsy was not feasible or when pathological results of biopsies were inconclusive with persistent suspicion of metastatic disease.

\section{Staging Laparoscopy (SL) and Exploratory Laparotomy}

SL was carried out by the HPB surgical fellow or surgeon and included inspection of the liver and gallbladder surface, peritoneal cavity, and hepatoduodenal ligament. ${ }^{5,11}$ Lymph node sampling was not performed routinely, but only for enlarged or suspicious lymph nodes. Furthermore, the lesser omentum was not routinely opened, but if done so, the common hepatic artery was inspected along its course, in search of suspicious lymph nodes. Laparoscopic ultrasound (LUS) was only performed in four patients within the study period.

Patients were scheduled for exploratory laparotomy when no metastases or locally advanced tumors were found at SL, when any new imaging did not show disease progression, and if the patient remained fit to undergo surgery. At laparotomy, the abdomen was inspected for any signs of incurable disease, such as peritoneal deposits, liver metastases, lymph node involvement beyond the hepatoduodenal ligament (N2), or locally advanced disease. Tumors were considered locally advanced if they invaded surrounding organs or when excessive vascular or biliary involvement precluded an $\mathrm{R} 0$ or $\mathrm{R} 1$ resection and only $\mathrm{R} 2$ resection was possible. Portal vein reconstructions were performed if necessary and technically feasible. All suspicious lesions and lymph nodes were biopsied and analyzed by frozen-section microscopic examination.

\section{Definition of Potential Risk Factors}

Study variables included clinical variables [jaundice, carbohydrate antigen (CA) 19-9] and radiological parameters that were retrospectively rereviewed on available computed tomography (CT) and magnetic resonance imaging (MRI) scans by an experienced staff radiologist (CYN) who was blinded to the outcome of SL.

Radiological parameters included BC classification, tumor size, vascular involvement, lobar atrophy, suspected lymph node metastases, and other suspicious metastatic lesions (intrahepatic, peritoneal). Suspicious lymph nodes were larger than $10 \mathrm{~mm}$ in short-axis diameter, had irregular border, or showed central necrosis and were categorized as perihilar (N1) or beyond the hepatoduodenal ligament (N2). ${ }^{10,12}$ Vascular involvement was defined as more than 180 degrees circumferential tumor contact or as clear distortion, narrowing or occlusion of the portal venous system and/or (branches of) hepatic artery. ${ }^{13}$ Lobar atrophy was defined as a small, hypoperfused lobe with crowding of dilated intrahepatic bile ducts. To avoid misinterpreting intrahepatic cholangiocarcinoma as PHC, we adhered to previously published anatomical landmarks. ${ }^{14}$

\section{Statistical Analysis}

The diagnostic accuracy of SL was expressed as the yield and sensitivity to detect incurable disease. The yield and sensitivity were calculated by dividing the total number of avoided laparotomies by the total number of laparoscopies and all patients with unresectable disease, respectively.

Logistic regression analysis was used to identify predictors of unresectable disease at SL. All study variables with a $p$ value $<0.100$ in univariable analysis were subsequently entered in multivariable analysis. A cut-off for tumor size was used in these analyses and determined at the highest sensitivity and specificity. A standard approach was then used to develop a risk score. ${ }^{15}$ Independent predictors ( $p<0.05$ in multivariable analysis) were selected and regression coefficients of these predictors were transformed into simple points to develop the risk score. The number of points assigned to each predictor equaled its regression coefficient in multivariable analysis divided by the 
predictor with the smallest absolute number of points, and rounded to the nearest whole number. Predicted risks were then calculated for each patient in the study cohort by applying total point scores to the logistic regression formula. Risk score tertiles were used to categorize patients into low-risk, intermediate-risk, and high-risk groups based on the predicted chance of finding metastases or locally advanced disease at SL.

Predictive accuracy (discrimination) of the risk score was assessed using area under the curve (AUC) analysis with $95 \%$ confidence intervals (CIs). The agreement between predicted and observed unresectability (calibration) was assessed using the Hosmer-Lemeshow goodnessof-fit test, with a significant outcome $(p<0.05)$ indicating a lack of fit. Missing data were rare and were handled with complete case analysis. All analyses were performed using IBM SPSS Statistics version 22.0 (IBM Corporation, Armonk, NY, USA) and R Version 3.1.2 (R Foundation for Statistical Computing, Vienna, Austria).

\section{RESULTS}

\section{Patient Demographics and Imaging}

A total of 656 patients with suspected PHC were identified within the study period. After multidisciplinary team discussion, 348 tumors were found to be potentially resectable and 273 patients underwent SL (Fig. 1). Preoperative staging was performed with ultrasound, CT in most patients (97\%), and more selectively with MRI (38\%) and positron emission tomography (PET; $22 \%$ ). Patient characteristics are presented in Table 1. Seventy-five patients were directly scheduled for exploratory laparotomy (electronic supplementary Table).

\section{Surgical Findings}

Inoperable tumors were found during SL in 41 patients, resulting in a yield of $15 \%$ (95\% CI 11-19). Lesions that were mainly detected were peritoneal and liver metastases. Twenty-nine patients who were considered resectable at SL did not undergo laparotomy (Fig. 1). After a median of 41 days (range 3-156), exploratory laparotomy was performed in 203 patients and showed unresectable disease in 83 patients $(41 \%)$, mainly because of lymph node metastases and locally advanced tumors that were not apparent on imaging or during SL (Fig. 1). SL correctly identified 41 of 136 patients with unresectable $\mathrm{PHC}$, resulting in an overall sensitivity of $30 \%$ (95\% CI 22-38). Highest sensitivity was found for peritoneal metastases $(27 / 37,73 \%)$, while sensitivity for detecting liver metastases was $39 \%(9 / 23)$. Locally advanced tumors $(8 / 55,15 \%)$ or lymph node metastases $(2 / 46,4 \%)$ were hardly detected by SL. Of all

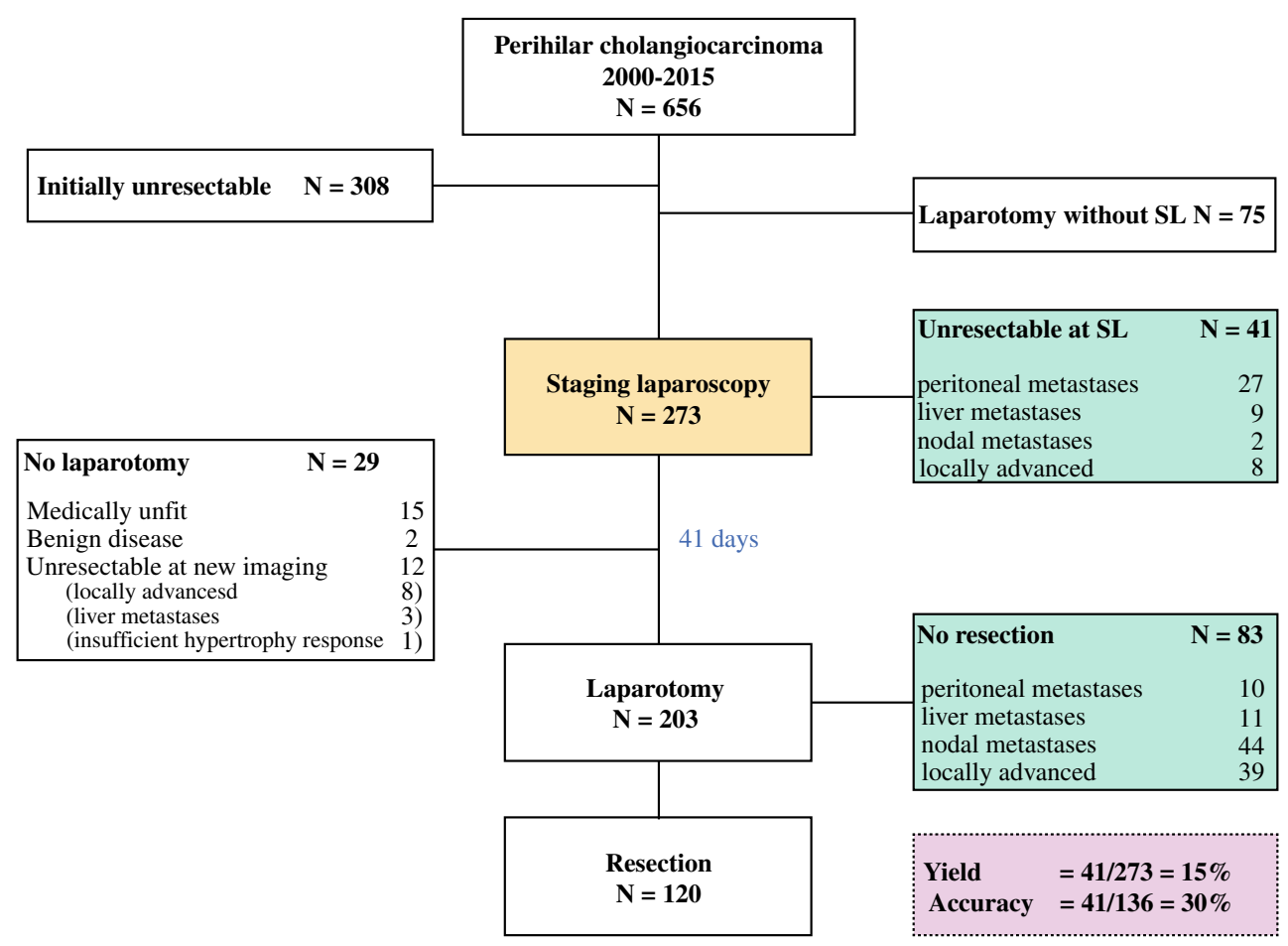

FIG. 1 Outcomes of patients with suspected perihilar cholangiocarcinoma undergoing staging laparoscopy and exploratory laparotomy at the Academic Medical Center between 2000 and 2015. SL staging laparoscopy 
TABLE 1 Baseline characteristics of the study cohort

\begin{tabular}{lc}
\hline & Patients $(n=273)$ \\
\hline Age, years [mean (SD)] & $65(11)$ \\
Jaundice at presentation & $235(86.1)$ \\
CA19-9, kU/L [median (range)] & $172(1-51,046)$ \\
Preoperative staging & \\
CT & $266(97.4)$ \\
MRI & $103(37.7)$ \\
US duplex & $73(26.7)$ \\
PET & $61(22.3)$ \\
Tumor size, cm [mean (SD)] & $2.8(1.3)$ \\
$\geq 4.5$ & $25(9.2)$ \\
Bismuth-Corlette type & \\
I & $12(4.4)$ \\
II & $20(7.3)$ \\
IIIa & $112(41.0)$ \\
IIIb & $60(22.0)$ \\
IV & $64(23.4)$ \\
Left or right duct & $5(1.8)$
\end{tabular}

Data are expressed as number of patients (\%), unless stated otherwise $S D$ standard deviation, CA19-9 carbohydrate antigen 19-9, CT computed tomography, MRI magnetic resonance imaging, US ultrasound, $P E T$ positron emission tomography

44 positive N2 lymph nodes that were found at laparotomy, 29 were located alongside the common hepatic artery.

Complications after SL occurred in eight patients (3\%) and were all minor (Clavien-Dindo grade I-II) and included urinary retention $(N=3)$, pneumonia $(N=1)$, pain requiring prolonged hospital stay $(N=1)$, PTC drain dislocation $(N=1)$, and fever requiring antibiotics $(N=2)$. Complications after exploratory laparotomy occurred in 27 patients (33\%) with unresectable disease, and included 10 major complications (including one death). Median hospital stay for SL was 3 days (range 1-9), including the day of admission.

\section{Preoperative Predictors of Unresectable Tumors at SL}

Univariable and multivariable analysis of predictors for detecting metastasized or locally advanced PHC at SL are shown in Table 2 . Independent predictors $(p<0.05$ in multivariable analysis) that were identified were tumor size $(\geq 4.5 \mathrm{~cm}$ ), portal vein involvement (bilateral or main stem), suspected metastases in N2 lymph nodes, and suspected (extra)hepatic metastases. No association was found for proximal extent of bile duct involvement (BC classification).

There was no difference in the yield of SL in patients who underwent MRI $(16 / 103,15.5 \%)$ compared with those who did not undergo MRI (25/170, $14.8 \%)$.

\section{Preoperative Risk Score}

The derived preoperative risk score to predict detection of metastasized or locally advanced PHC at SL is presented in Table 3. The sum of the risk score ranges between 0 and 5 points, and predicted risks for each point score are presented in Table 4. The predicted risk was $7.2 \%$ in the low-risk tertile ( 0 points, $N=203$ patients), $21.3 \%$ in the intermediate-risk tertile (1 point, $N=39$ ), and $58.0 \%$ (range $48.5-91.9 \%$ ) in the high-risk tertile ( $\geq 2$ points, $N=31$ ).

Predictive performance of the preoperative risk score was well, with an AUC of 0.77 (95\% CI 0.68-0.86) and excellent calibration was observed (Hosmer-Lemeshow test $p=0.995$ ). Predictive accuracy remained good after categorizing patients into low-risk, intermediate-risk, and high-risk groups (AUC 0.77, $95 \%$ CI 0.68-0.86).

\section{DISCUSSION}

This is the largest study reporting on the use of SL in PHC. In 273 consecutive patients, a relatively low yield $(15 \%)$ and overall sensitivity (30\%) of SL were found to detect unresectable PHC. Several independent risk factors were identified that accurately predicted detection of metastases or locally advanced tumors. A preoperative risk score was developed that showed good discrimination to predict unresectable PHC at SL.

Few reports have studied the additional role of laparoscopy in preoperative staging of PHC, with varying results. ${ }^{6,9,16-18}$ Remarkably, until now only one study was able to identify any predictors for a positive yield. ${ }^{18}$ In that study, a significantly higher yield was found for tumors classified as $\mathrm{T} 2$ and $\mathrm{T} 3$, rather than $\mathrm{T} 1$, according to the Blumgart staging system. This observation was not confirmed in a recent study including 100 patients, but reevaluation of scans and $\mathrm{T}$ staging had been performed in only 38 patients in that cohort. ${ }^{9}$ Notably, only one of three criteria of the Blumgart system (i.e. PV involvement) was associated with unresectability at SL in our analysis. However, the Blumgart staging system was originally developed for predicting resectability in a complete cohort of patients with PHC, and may therefore be less applicable for the decision to perform SL. Variables that were identified as independent predictors in our study and that were included in the risk score were tumor size of $4.5 \mathrm{~cm}$ or more, bilateral PV involvement, suspicious lymph node metastases, and suspicious (extra)hepatic metastases on imaging. Suspected metastases involved lesions in which it was not possible to obtain histology by percutaneous approach or when there was an inconclusive biopsy in spite of persistent suspicion. The extent of bile duct involvement was not associated with unresectability at SL, therefore 
TABLE 2 Univariable and multivariable analysis of risk factors for detecting unresectable perihilar cholangiocarcinoma at staging laparoscopy

\begin{tabular}{|c|c|c|c|c|c|}
\hline \multirow[t]{2}{*}{ Variable } & \multirow{2}{*}{$\begin{array}{l}\text { Patients } \\
{[n(\%)]}\end{array}$} & \multicolumn{2}{|c|}{ Univariable analysis } & \multicolumn{2}{|c|}{ Multivariable analysis } \\
\hline & & OR $(95 \% \mathrm{CI})$ & $p$ value & OR $(95 \% \mathrm{CI})$ & $p$ value \\
\hline Jaundice at presentation & $235(86.1)$ & $1.6(0.5-4.7)$ & 0.407 & - & \\
\hline CA19-9 & - & $1.0(1.0-1.0)$ & 0.764 & - & \\
\hline Bismuth-Corlette type & & & & - & \\
\hline I & $12(4.4)$ & Reference & & & \\
\hline II & $20(7.3)$ & $1.7(0.3-10.3)$ & 0.583 & & \\
\hline IIIa & $112(41.0)$ & $0.7(0.1-3.3)$ & 0.612 & & \\
\hline IIIb & $60(22.0)$ & $0.6(0.1-3.2)$ & 0.507 & & \\
\hline IV & $64(23.4)$ & $1.3(0.3-6.5)$ & 0.771 & & \\
\hline Left or right duct & $5(1.8)$ & $3.3(0.3-34.8)$ & 0.315 & & \\
\hline \multicolumn{6}{|l|}{ Tumor size, $\mathrm{cm}$} \\
\hline$<4.5$ & $248(90.8)$ & Reference & & & \\
\hline$\geq 4.5$ & $25(9.2)$ & $3.8(1.6-9.3)$ & 0.004 & $4.1(1.4-11.8)$ & 0.008 \\
\hline \multicolumn{6}{|l|}{ Portal vein involvement } \\
\hline None & $151(55.3)$ & Reference & & & \\
\hline Unilateral & $98(35.9)$ & $1.1(0.5-2.4)$ & 0.754 & $1.1(0.5-2.7)$ & 0.830 \\
\hline Bilateral or main stem & $24(8.8)$ & $5.3(2.0-13.6)$ & 0.001 & $3.9(1.3-12.2)$ & 0.021 \\
\hline \multicolumn{6}{|l|}{ Hepatic artery involvement } \\
\hline None & $175(64.1)$ & Reference & & & \\
\hline Unilateral & $89(32.6)$ & $1.5(0.7-3.1)$ & 0.279 & $1.3(0.5-3.0)$ & 0.564 \\
\hline Bilateral or main stem & $9(3.3)$ & $9.2(2.3-36.9)$ & 0.002 & $4.2(0.7-23.4)$ & 0.105 \\
\hline \multicolumn{6}{|l|}{ Suspected lymph node metastases } \\
\hline None & $181(66.3)$ & Reference & & & \\
\hline N1 & $73(26.7)$ & $1.2(0.5-2.7)$ & 0.645 & $0.6(0.2-1.7)$ & 0.345 \\
\hline $\mathrm{N} 2$ & $19(7.0)$ & $8.5(3.1-23.2)$ & $<0.001$ & $4.9(1.4-16.6)$ & 0.012 \\
\hline Suspected (extra)hepatic metastases & $22(8.1)$ & $9.2(3.7-23.2)$ & $<0.001$ & $9.3(2.9-30.4)$ & $<0.001$ \\
\hline Lobar atrophy & $63(23.1)$ & $0.8(0.3-1.8)$ & 0.558 & - & \\
\hline
\end{tabular}

Suspected metastases were suspicious metastatic lesions on imaging for which diagnosis by percutaneous biopsy was not feasible or when pathological results of biopsies were inconclusive with ongoing suspicion of metastases. N2 lymph nodes were located beyond the hepatoduodenal ligament

OR odds ratio, CI confidence interval, CA19-9 carbohydrate antigen 19-9

TABLE 3 Preoperative risk score to predict unresectable perihilar cholangiocarcinoma at staging laparoscopy

\begin{tabular}{lll}
\hline Variable & Classes & Points \\
\hline Tumor size, cm & $<4.5$ & 0 \\
& $\geq 4.5$ & 1 \\
Portal vein involvement & None or unilateral & 0 \\
& Bilateral or main stem & 1 \\
Suspected lymph node metastases & None or N1 lymph & 0 \\
& nodes & \\
Suspected (extra)hepatic & N2 lymph nodes & 1 \\
metastases $^{\mathrm{a}}$ & No & 0 \\
& Yes & 2 \\
\hline
\end{tabular}

${ }^{a}$ Ongoing suspicion on lymph node or (extra)hepatic metastases after previous inconclusive/negative biopsy selecting patients for SL based on the BC classification does not seem justified.

In this study, SL had the highest sensitivity for detecting peritoneal metastases, whereas sensitivity for liver metastases, locally advanced tumors, and lymph node metastases was poor. A higher incidence of peritoneal and liver metastases was noted in large tumors and tumors with bilateral PV involvement compared with those without these criteria. In addition, among patients in whom N2 lymph node metastases were suspected on preoperative imaging, a substantial proportion had peritoneal metastases at SL. The reasons for missed superficial liver metastases in this study are not entirely clear as most lesions at laparotomy were located in the anterior liver segments and therefore could have potentially been detected at SL. 
TABLE 4 Predicted and observed risks according to the risk score points

\begin{tabular}{llrll}
\hline Group & Point total & $N$ & \multicolumn{2}{c}{ Unresectability at SL } \\
\cline { 4 - 5 } & & & Predicted (\%) & Observed (\%) \\
\hline Low-risk & 0 & 203 & 7.2 & 6.4 \\
Intermediate-risk & 1 & 39 & 21.3 & 28.2 \\
High-risk & 2 & 21 & 48.5 & 47.6 \\
& 3 & 9 & 76.5 & 66.7 \\
& 4 & 1 & 91.9 & 100 \\
& 5 & 0 & NA & NA \\
\hline
\end{tabular}

$N$ number of patients in the study for each point score, $N A$ not applicable, $S L$ staging laparoscopy

The median interval between SL and laparotomy was 41 days and included required time for adequate biliary drainage and/or hypertrophy to occur after portal vein embolization. Metastases may have developed or may have grown substantially to become visible in that time, although PHC is characterized by a rather indolent growth. Ideally, SL and laparotomy would be performed in a single session in order to limit hospital admissions and surgical procedures. However, diagnosis of malignancy is sometimes difficult on frozen sections and biopsy material may require additional (immunohistochemical) staining. In addition, many centers face logistical issues related to anesthetic and operating room time planning, and prefer to perform SL separately from laparotomy. Furthermore, early detection of inoperability allows the timely start of palliative care.

At laparotomy, N2 lymph node metastases were a common finding precluding resection, of which the majority were located along the course of the common hepatic artery. As others confirm the high incidence of unexpected lymph node metastases at laparotomy, ${ }^{2}$ a more extended laparoscopic staging procedure in high-risk patients is recommended. Instead of only visualizing the liver surface and peritoneal cavity, routine opening of the lesser sac, and biopsy of lymph nodes at the anterior side of the common hepatic artery (lymph node station 8a), is currently added in these patients in our department. The incidence of complications following SL was low in this study and included only minor events. However, $20 \%$ minor and $12 \%$ major complications (including one death) were observed following exploratory laparotomy in patients with unresectable PHC. These findings highlight the need for accurate preoperative staging.

LUS was only performed in four patients in this study period as it was previously found to be of limited value in our center. ${ }^{11}$ LUS may theoretically be useful in detecting non-superficial liver metastases or vascular involvement, but obtaining histological evidence in the latter case might be difficult without exploration of the liver hilum. Unfortunately, limited data on the additional value of LUS are available from other studies in the literature. ${ }^{4}$ In only two of six studies, the diagnostic yield of SL was increased with the use of LUS, mainly due to the identification of locally advanced tumors. ${ }^{16,19}$

The eventual yield of SL is influenced by the extent of preoperative imaging. In our cohort, most patients underwent CT, whereas MRI and PET-CT were only performed in selected patients. Limited data are available to assume that the additional use of MRI will increase the yield of imaging in potentially resectable PHC. Previous studies have found no differences in the diagnostic accuracy of CT or MRI for detecting lymph node metastases. ${ }^{20-22}$ Nonetheless, studies are hampered by their retrospective design and small sample size. The additional value of PET-CT was previously investigated at our center, but all patients had undergone SL in that study. ${ }^{23}$ It was found that the yield of PET-CT after staging using CT and laparoscopy was disappointing because of the low sensitivity for detecting distant metastases and comparable detection of lymph node metastases. However, in a large, prospective study including patients not undergoing SL, it was shown that PET-CT had higher accuracy over CT/MRI in the detection of lymph node metastases and distant metastases. ${ }^{24}$ Furthermore, in that study it was shown that PETCT revealed unsuspected metastases and clarified indeterminate lesions. Future studies might compare the use of PET-CT and SL in the preoperative staging of PHC. Lastly, EUS is often performed to evaluate suspicious lymph nodes. Although the results of EUS-guided lymph node biopsy vary in the literature, this less-invasive technique seems a preferred step prior to $\mathrm{SL} ;{ }^{25}$ however, some caution may be advised as tumor tract seeding has been reported. ${ }^{26}$

The present study has several limitations. The relatively few unresectable tumors that were detected at SL consequently provided a low number of events for statistical analysis, which may have led to statistical uncertainty in the multivariable analysis, as reflected by the relatively wide CIs. Second, we were unable to perform external validation of the proposed risk score because of the limited sample size. Future studies that validate the risk score are therefore desirable. Third, as this study comprises a cohort of 15 years, the quality of preoperative imaging varied during the study period and may have subsequently influenced the assessment of vascular involvement or metastases in the early years. However, scans were rereviewed by a specialized radiologist and we also did not note any changes in the diagnostic yield of SL within the study period.

\section{CONCLUSIONS}

The results from this study support a selective approach to SL in patients with potentially resectable PHC. The proposed 
preoperative risk score can be useful in selecting patients who may benefit most from this additional staging procedure. Patients in the low-risk group can proceed to exploratory laparotomy without SL. The use of SL in the intermediate-risk group is debatable, whereas patients in the high-risk group are likely to be diagnosed with incurable disease at SL, thereby avoiding an unnecessary laparotomy.

DISCLOSURES Robert J.S. Coelen received a travel grant from the United European Gastroenterology (UEG) Week for best submitted abstract, and for presentation of this work at the UEG Week Congress held in Barcelona, Spain, in October 2015.

OPEN ACCESS This article is distributed under the terms of the Creative Commons Attribution 4.0 International License (http:// creativecommons.org/licenses/by/4.0/), which permits unrestricted use, distribution, and reproduction in any medium, provided you give appropriate credit to the original author(s) and the source, provide a link to the Creative Commons license, and indicate if changes were made.

\section{REFERENCES}

1. Ito F, Cho CS, Rikkers LF, Weber SM. Hilar cholangiocarcinoma: current management. Ann Surg. 2009;250:210-8.

2. Matsuo K, Rocha FG, Ito K, D'Angelica MI, Allen PJ, Fong Y, et al. The Blumgart preoperative staging system for hilar cholangiocarcinoma: analysis of resectability and outcomes in 380 patients. J Am Coll Surg. 2012;215:343-5.

3. Ruys AT, van Haelst S, Busch OR, Rauws EA, Gouma DJ, van Gulik TM. Long-term survival in hilar cholangiocarcinoma also possible in unresectable patients. World J Surg. 2012;36:2179-86.

4. Coelen RJ, Ruys AT, Besselink MG, Busch OR, van Gulik TM. Diagnostic accuracy of staging laparoscopy for detecting metastasized or locally advanced perihilar cholangiocarcinoma: a systematic review and meta-analysis. Surg Endosc. doi:10.1007/ s00464-016-4788-y

5. Ruys AT, Busch OR, Gouma DJ, van Gulik TM. Staging laparoscopy for hilar cholangiocarcinoma: is it still worthwhile? Ann Surg Oncol. 2011;18:2647-53.

6. Russolillo N, D'Eletto M, Langella S, Perotti S, Lo Tesoriere R, Forchino F, et al. Role of laparoscopic ultrasound during diagnostic laparoscopy for proximal biliary cancers: a single series of 100 patients. Surg Endosc. 2016;30(3):1212-8.

7. Ratti F, Cipriani F, Ferla F, Catena M, Paganelli M, Aldrighetti LA. Hilar cholangiocarcinoma: preoperative liver optimization with multidisciplinary approach. Toward a better outcome. World J Surg. 2013;37:1388-96.

8. Gomez D, Patel PB, Lacasia-Purroy C, Byrne C, Sturgess RP, Palmer D, et al. Impact of specialized multi-disciplinary approach and an integrated pathway on outcomes in hilar cholangiocarcinoma. Eur J Surg Oncol. 2014;40:77-84.

9. Barlow AD, Garcea G, Berry DP, Rajesh A, Patel R, Metcalfe MS, et al. Staging laparoscopy for hilar cholangiocarcinoma in 100 patients. Langenbecks Arch Surg. 2013;398:983-8.

10. Edge SB Byrd DR, Compton CC, Fritz AG, Greene FL, Trotti A, editors. AJCC cancer staging manual. 7th ed. New York: Springer; 2010.
11. Tilleman EH, de Castro SM, Busch OR, Bemelman WA, van Gulik TM, Obertop H, et al. Diagnostic laparoscopy and laparoscopic ultrasound for staging of patients with malignant proximal bile duct obstruction. J Gastrointest Surg. 2002;6:426-30; discussion 430-421.

12. Lee HY, Kim SH, Lee JM, Kim SW, Jang JY, Han JK, et al. Preoperative assessment of resectability of hepatic hilar cholangiocarcinoma: combined CT and cholangiography with revised criteria. Radiology. 2006;239:113-21.

13. Jarnagin WR, Fong Y, DeMatteo RP, Gonen M, Burke EC, Bodniewicz BJ, et al. Staging, resectability, and outcome in 225 patients with hilar cholangiocarcinoma. Ann Surg. 2001;234:507-517; discussion 517-509.

14. Ebata T, Kosuge T, Hirano S, Unno M, Yamamoto M, Miyazaki $\mathrm{M}$, et al. Proposal to modify the International Union Against Cancer staging system for perihilar cholangiocarcinomas. $\mathrm{Br} \mathrm{J}$ Surg. 2014;101:79-88.

15. Sullivan LM, Massaro JM, D'Agostino RB Sr. Presentation of multivariate data for clinical use: The Framingham Study risk score functions. Stat Med. 2004;23:1631-60.

16. Connor S, Barron E, Wigmore SJ, Madhavan KK, Parks RW, Garden OJ. The utility of laparoscopic assessment in the preoperative staging of suspected hilar cholangiocarcinoma. $J$ Gastrointest Surg. 2005;9:476-80.

17. Goere D, Wagholikar GD, Pessaux P, Carrere N, Sibert A, Vilgrain $\mathrm{V}$, et al. Utility of staging laparoscopy in subsets of biliary cancers: laparoscopy is a powerful diagnostic tool in patients with intrahepatic and gallbladder carcinoma. Surg Endosc. 2006;20:721-25.

18. Weber SM, DeMatteo RP, Fong Y, Blumgart LH, Jarnagin WR. Staging laparoscopy in patients with extrahepatic biliary carcinoma. Analysis of 100 patients. Ann Surg. 2002;235:392-99.

19. Vollmer CM, Drebin JA, Middleton WD, Teefey SA, Linehan DC, Soper NJ, et al. Utility of staging laparoscopy in subsets of peripancreatic and biliary malignancies. Ann Surg. 2002;235:1-7.

20. Park HS, Lee JM, Choi JY, Lee MW, Kim HJ, Han JK, et al. Preoperative evaluation of bile duct cancer: MRI combined with MR cholangiopancreatography versus MDCT with direct cholangiography. AJR Am J Roentgenol. 2008;190:396-405.

21. Ruys AT, van Beem BE, Engelbrecht MR, Bipat S, Stoker J, Van Gulik TM. Radiological staging in patients with hilar cholangiocarcinoma: a systematic review and meta-analysis. $\mathrm{Br} \mathrm{J}$ Radiol. 2012;85:1255-62.

22. Zhang H, Zhu J, Ke F, Weng M, Wu X, Li M, et al. Radiological imaging for assessing the respectability of hilar cholangiocarcinoma: a systematic review and meta-analysis. Biomed Res Int. 2015;2015:497942.

23. Ruys AT, Bennink RJ, van Westreenen HL, Engelbrecht MR, Busch OR, Gouma DJ, et al. FDG-positron emission tomography/computed tomography and standardized uptake value in the primary diagnosis and staging of hilar cholangiocarcinoma. $H P B$ (Oxford). 2011;13:256-62.

24. Kim JY, Kim MH, Lee TY, Hwang CY, Kim JS, Yun SC, et al. Clinical role of 18F-FDG PET-CT in suspected and potentially operable cholangiocarcinoma: a prospective study compared with conventional imaging. Am J Gastroenterol. 2008;103:1145-51.

25. Strongin A, Singh H, Eloubeidi MA, Siddiqui AA. Role of endoscopic ultrasonography in the evaluation of extrahepatic cholangiocarcinoma. Endosc Ultrasound. 2013;2:71-6.

26. Heimbach JK, Sanchez W, Rosen CB, Gores GJ. Trans-peritoneal fine needle aspiration biopsy of hilar cholangiocarcinoma is associated with disease dissemination. HPB (Oxford). 2011;13:356-60. 